\title{
Acciones socioeducativas sobre sexualidad para la familia de infantes con parálisis cerebral en el Centro Internacional de Restauración Neurológica
}

\author{
Socio-educational Actions on Sexuality for the Family of Infants with Cerebral Palsy at the Interna- \\ tional Center of Neurological Restoration
}

\author{
Grethel Ortiz-Antón ${ }^{1 *}$ \\ 1 Centro Internacional de Restauración Neurológica CIREN. La Habana, Cuba.
}

\section{Resumen}

Esta investigación tiene como objetivo la propuesta de acciones socioeducativas dirigida a las madres y padres de infantes diagnosticados con parálisis cerebral, que asistieron a la Clínica de Atención a Pacientes Cubanos del CIREN en el periodo de mayo de 2009 a febrero de 2010 para recibir el Programa de Rehabilitación Multifactorial Intensiva. Se efectuó un estudio descriptivo de corte transversal, con una perspectiva cualitativa, que determinó la percepción de madres y padres acerca de la sexualidad de sus hijas/os. Se aplicó una encuesta y se utilizó la técnica cualitativa de entrevista en profundidad para realizar el estudio. El diagnóstico demostró que existen vacíos en el conocimiento en la esfera de la sexualidad desde el rol de madres y padres, limitándose el conocimiento sobre esta temática permeado de mitos y prejuicios. No es suficiente la información recibida sobre los temas de la sexualidad infantil y consideran que la educación sexual de niñas/os que presentan alguna discapacidad puede ser necesaria si se condiciona a la recuperación física. En tanto, expresan que la sociedad no está preparada para convivir con la diferencia. La elaboración de las acciones socioeducativas se fundamentó a partir de la información obtenida en el proceso diagnóstico.

Palabras clave: acciones socioeducativas, sexualidad, familia de infantes, parálisis cerebral

\section{Abstract}

This research summary aims at proposing correctional actions addressed to mothers and fathers of children diagnosed with Cerebral Palsy who attended the clinic attention to the CIREN Cuban patients during May 2009 to February 2010 to receive the Multifactorial intensive rehabilitation program. A descriptive study of cross-section, with a qualitative perspective that led to the perception of parents about the sexuality of his daughters was made / I. It applied a survey and qualitative interview technique was used in depth for the study. The diagnosis proved that they exist gaps in knowledge in the field of sexuality from the role of mothers and fathers, limiting the knowledge on this topic permeated myths and prejudices. The information received is not enough on the issues of child sexuality and consider that sex education of girls / boys who have a disability may be necessary if the physical recovery is conditioned. While they express that society is not prepared to live with difference. The elaboration of socio-educational actions was based on the information obtained in the diagnostic process.

Keywords: socio-educational actions, sexuality, family of infants, cerebral palsy

\footnotetext{
*Correspondencia: gortiz@neuro.ciren.cu, cineuro@neuro.ciren.cu. Centro Internacional de Restauración Neurológica CIREN. Avenida 25 No. 15805 Cubanacán, Playa. Ciudad de La Habana, Cuba. Teléfono: +53 (7) 2 736 087, 273677 778. Fax: +53 (7) 2736302,2732420 Recibido: 29-10-11. Revisión desde: 30-11-11. Aceptado: 23-12-11

DOI: $10.5839 / \mathrm{rcnp} .2011 .0602 .08$
} 


\section{Introducción}

La sexualidad es una manifestación vital de la personalidad que permite la expresión individual y social que cada sujeto ha podido interiorizar a través de la actividad misma y la comunicación que la fundamenta. Se matiza por el carácter individualizado y la condición histórica social que la contextualiza.

Como una compleja y rica manifestación humana, la sexualidad se construye, se vivencia y expresa en todas las dimensiones existenciales: el individuo, la pareja, la familia y la sociedad. En el individuo se integra el yo íntimo, proyectándose desde su masculinidad o feminidad, mientras que el comportamiento con la pareja, la familia y la sociedad le imprimen un contenido social a esta importante manifestación existencial de la personalidad, estableciéndose vínculos afectivos y eróticos en la comunicación física y espiritual (González y Castellanos, 2003).

En nuestra sociedad, las personas con discapacidad constituyen un grupo poblacional importante. Frecuentemente se enfrentan a un sinnúmero de restricciones para su desenvolvimiento social, no solo por las limitaciones físicas o mentales, sino por actitudes y conductas inapropiadas de otros individuos. Se hace más evidente cuando de la sexualidad se trata, ya que existen numerosos prejuicios, tabúes y estigmas que les impiden expresar y vivenciar adecuadamente este aspecto fundamental de la existencia humana.

La sexualidad no se vivencia igualmente si la afectación ocurre antes o después de la adolescencia; además, la falta de pareja y los problemas sexuales resultan ser más frecuentes entre los menores con discapacidades desde edades tempranas. Las actividades sexuales solitarias se realizan menos que lo esperado en los individuos con discapacidad desde estas edades (Gil Almira y Cardero Pacheco, 2003), circunstancia que podría estar determinada por la falta de una educación y orientación sexual apropiada.

En el mundo la tendencia investigativa relacionada con los temas de la sexualidad y la discapacidad no ocupan el porcentaje más significativo; sin embargo, existen estudios que fortalecen la temática por los resultados que benefician a esta población con derechos sexuales. En países como España, Estados Unidos, Chile y Argentina se han desarrollado proyectos para la inclusión de los asuntos de la sexualidad y la discapacidad. Sobre la familia y la sexualidad de discapacitados se han desarrollado internacionalmente cursos de preparación, manuales, libros y otras alternativas que facilitan la obtención de información y acción. Se vinculan frecuentemente los temas de la sexualidad infantil con los temas del abuso sexual, entre otras manifestaciones de la violencia.

En Cuba se han desarrollado desde hace más de una década proyectos de intervención dirigidos a la sexualidad de las personas que presentan alguna discapacidad, a la familia de niñas y niños diagnosticados con alguna patología discapacitante, a las educadoras/es especiales y otros profesionales de la educación y la salud pública.

La influencia de las madres y los padres no siempre se muestra apropiada, bien por falta de información o por prejuicios y estereotipos al respecto. Ampliar el conocimiento y desempeño de los familiares para ayudar a que los infantes con discapacidades desarrollen una sexualidad sana desde sus perspectivas individuales, contribuirá al desarrollo de esta dimensión de la personalidad.
La construcción de valores que comienza desde los primeros años de vida constituye un proceso de formación y desarrollo necesario para las niñas y los niños. El acompañamiento en esta etapa por las figuras materna y paterna va a favorecer la adquisición de conocimientos, destrezas y habilidades, no solo para beneficiar la esfera cognoscitiva y la socialización como proceso integrador con el medio que les rodea, sino también para apropiar elementos imprescindibles para la educación de la sexualidad infantil, tales como los autocuidados sistemáticos y la autoestima positiva para obtener afrontamientos enfocados hacia un desarrollo pleno.

En el trabajo cotidiano durante el proceso de neurorestauración no es común escuchar a las madres y los padres abordando el tema de la sexualidad de sus hijos; sin embargo, durante el diálogo con ellos emergen las preocupaciones acerca del manejo de la sexualidad infantil. Otros expresan rechazo por el tema porque temen incentivar "conductas desviadas en los niños" o "que se conviertan en objeto de abuso sexual por no cumplir con las expectativas sociales relacionadas con el aspecto físico".

En un estudio realizado en 2006 por el Dr. Castillo, se llega a la conclusión que cuando no se posee un conocimiento completo y sostenido sobre lo que ocurre con la sexualidad de las personas con discapacidad, no es posible crear estrategias para la educación, orientación y tratamiento sexual, con vistas a remediar los posibles problemas que surjan en esta esfera tan importante de la vida. Es frecuente que los padres, e incluso profesionales de la rehabilitación, tengan una visión muy limitada sobre la sexualidad, al vincularla con la respuesta sexual biológica, el estado físico de los niños y la capacidad de formar pareja erótica.

Abordar la temática de la sexualidad y la discapacidad infantil constituye un reto importante para los profesionales que trabajan la neurorestauración. Investigar y prescribir alternativas que beneficien el desarrollo de la sexualidad de aquellas personas que presentan alguna discapacidad puede favorecer un mayor alcance en el tratamiento multifactorial intensivo.

La puesta en marcha de las acciones socioeducativas, que por primera vez se realizan en el Centro, propiciará el enriquecimiento del conocimiento sobre temas de la sexualidad infantil para madres y padres, lo cual contribuirá al desarrollo personal de sus hijas e hijos. La implementación de acciones socioeducativas ofrecerá a las madres y padres de infantes con parálisis cerebral herramientas educativas para la sistematización de conocimientos específicos sobre la sexualidad infantil.

\section{Material y método}

La presente investigación se desarrolló en el periodo de mayo de 2009 a febrero de 2010. Se realizó un diagnóstico a partir de la identificación de las necesidades básicas de aprendizaje sobre temas de la sexualidad infantil de la muestra estudiada. Posteriormente se elaboró la propuesta de acciones socioeducativas sobre temas de la sexualidad para las madres y los padres cuyos hijos/as presentan un diagnóstico de parálisis cerebral.

\section{Objetivo general}

- Diseñar un plan de acciones socioeducativas que contribuya a la educación sobre temas de la sexualidad a las madres y los padres de infantes con parálisis cerebral que asisten al CIREN. 
Objetivos específicos

- Identificar la percepción que poseen las madres y los padres sobre temas de la sexualidad infantil.

- Diagnosticar las necesidades básicas de aprendizaje relacionadas con la sexualidad infantil del grupo estudiado.

Problema científico: ¿Cómo contribuir a la educación sobre los temas de la sexualidad infantil a las madres y los padres de infantes con parálisis cerebral que asisten al CIREN en el periodo de mayo de 2009 a febrero de 2010?

Perspectiva metodológica: Cualitativa.

En el estudio se utiliza una metodología cualitativa, con perspectiva fenomenológica, que permite analizar los valores, ritos y significados, recoge lo social vivido y su sentido e impacto en el grupo estudiado. Nos facilita entender situaciones, interpretar fenómenos y desarrollar conceptos, haciendo énfasis en las experiencias y las opiniones de los participantes. Este tipo de investigación trata de identificar la naturaleza profunda de las realidades y su estructura dinámica, dando razón plena de su comportamiento y manifestaciones. Se orienta a determinar el significado del fenómeno, permitiendo conocer la percepción que los sujetos tienen de su realidad (Rodríguez Gómez et al., 2002).

Se utilizó el método IAP (investigación acción participativa), con el propósito de construir la propuesta de acciones socioeducativas a partir de un diagnóstico participativo.

Este método de investigación-acción permite que los sujetos investigados participen como coinvestigadores en todas las fases del proceso: planteamiento del problema, recolección de la información e interpretación de la misma, planeación y ejecución de la acción concreta para la solución del problema y evaluación posterior sobre lo realizado. El fin principal de estas investigaciones no es algo exógeno a las mismas, sino que está orientado hacia la concientización, desarrollo y emancipación de los grupos estudiados y hacia la solución de sus problemas (Martínez, 2006).

En esta perspectiva, se combina la investigación social, el trabajo educativo y la acción transformadora, implicando un proceso necesario de empoderamiento social e individual a través del desarrollo de habilidades y capacidades para la toma de decisiones, en la solución de necesidades básicas de aprendizaje sobre temas de sexualidad infantil.

Tipo de investigación: Descriptiva de corte transversal. Diseño de investigación: Casos múltiples.

La población estuvo conformada por todas las madres y padres cuyos hijos/as reciben el Programa Multifactorial Intensivo de Neuro-rehabilitación en la Clínica de Atención a Pacientes Cubanos del CIREN. Se realiza una selección muestral intencional de sujetos tipo. La muestra quedó constituida por 18 madres y 9 padres que asistieron con sus hijas/os diagnosticados con parálisis cerebral en el periodo de mayo de 2009 a febrero de 2010 a la Clínica de Atención a Pacientes Cubanos del CIREN.

\section{Métodos y técnicas}

Del nivel teórico se utilizó el método histórico lógico, con el propósito de establecer una línea de evolución histórica con un orden lógico sobre teorías, posiciones y enfoques sobre los temas de sexualidad, discapacidad, infancia y familia.

Del nivel empírico se hizo uso de la observación científica, para lo cual se elaboró una guía de observación (ver tabla 1) que sirvió de pauta para el análisis y la evaluación de esta problemática social, permitiendo la comprensión del significado de cada narración durante el proceso de interacción, así como reconocer el vínculo que establecen los niños y niñas en el contexto de la rehabilitación con sus padres y terapeutas. Se prestó especial atención a la expresión de las emociones, los gestos y los silencios.

Para el procesamiento de la información se utilizó el análisis de contenido, que permitió realizar una lectura e interpretación discursiva de los resultados obtenidos. El análisis de contenido ofrece la posibilidad de investigar sobre la naturaleza del discurso, admitiendo examinar con detalle y profundidad cada elemento contenido en la comunicación humana.

Del nivel empírico se aplicó un cuestionario para indagar la visión sobre la sexualidad que poseían las madres que acompañaban a sus hijas/os. Esta técnica permitió abordar el tema desde una óptica exploratoria al inicio de la investigación. Proporciona la posibilidad de recoger información, lo que facilita la concreción de supuestos, creencias o modelos de partida usados para explicar una determinada realidad. Se utilizó la encuesta que empleó la Dra. Lissette Torres Marrero, en su tesis: "Visión materna de la sexualidad de niños con Parálisis Cerebral", en 2004 (anexo 2).

Después de aplicar el cuestionario a las madres, emergieron demandas de los padres que asistieron de forma regular al ciclo de rehabilitación con sus hijos/as. Expresaron el deseo de colaborar con la investigación y fueron incluidos en la muestra estudiada.

Tabla 1. Guía de Observación.

\begin{tabular}{|c|c|c|}
\hline Topico & & Elementos \\
\hline $\begin{array}{l}\text { Persona a quien } \\
\text { observa } \\
\text { Tipo de observación } \\
\text { Condiciones en que } \\
\text { realiza la observación }\end{array}$ & se & $\begin{array}{l}\text { Madres y padres objeto de estudio } \\
\text { Directa, no sistematizada. } \\
\text { En el CIREN - campo de acción. }\end{array}$ \\
\hline $\begin{array}{l}\text { Objetivos } \\
\text { observación }\end{array}$ & la & $\begin{array}{l}\text { - Recoger información relacionada con el vínculo que } \\
\text { establecen las madres y los padres con sus hijas/os y } \\
\text { con las/os terapeutas. } \\
\text { - Recoger las manifestaciones de la conducta durante } \\
\text { el desarrollo de la investigación, teniendo en cuenta } \\
\text { la expresión de las emociones, los gestos, } \\
\text { expresiones corporales y los silencios. } \\
\text { - Recoger las expresiones asociadas al tema ofrecidas } \\
\text { por las madres y los padres en los diferentes } \\
\text { contextos de trabajo. }\end{array}$ \\
\hline $\begin{array}{l}\text { Intereses para } \\
\text { observación. }\end{array}$ & la & $\begin{array}{l}\text { - Cuáles son las características de la comunicación (si } \\
\text { es clara y directa en relación con el tema que se esté } \\
\text { trabajando). } \\
\text { - Cuáles son las expresiones que se utilizan según } \\
\text { temas (estereotipos, prejuicios, símbolos, códigos, } \\
\text { percepciones, representaciones que usan asociadas a } \\
\text { los temas que se trabajan). }\end{array}$ \\
\hline
\end{tabular}

Tabla 2. Modalidad para explorar temas de sexualidad

Guía de entrevista en profundidad

- Criterios de las madres y padres acerca de la sexualidad

- Ambiente escolar y sociocultural como facilitador de la educación sexual - Composición y relaciones intrafamiliares

- Términos que utilizan madres y padres para abordar el tema de la sexualidad

- Preocupaciones fundamentales de madres y padres relacionadas con la sexualidad de sus hijos/as.

- Valoración de situaciones existenciales 
Realizamos esta encuesta anónima a las madres de las niñas y los niños que se diagnostican con una parálisis cerebral para que sus resultados nos faciliten mejorar la atención de la esfera de la sexualidad de las niñas y los niños. Si Ud. no se siente en condiciones para responder con sinceridad las preguntas, entonces comuníquelo a la encuestadora. Si siente la necesidad de ser orientada o realizar alguna consulta, puede transmitírselo a la encuestadora.

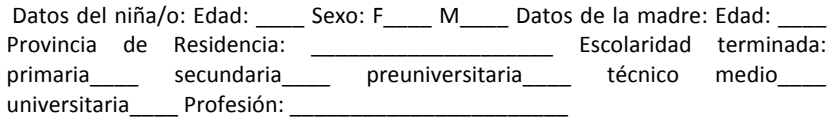

1. Cree que su niña/o pueda desarrollar una sexualidad gratificante en su adultez: Si__No___ ¿Por qué no?

2. Para que la niña/o desarrolle plenamente su sexualidad es necesario: Realizarle el máximo de ejercicios para que mejore sus condiciones físicas. Transmitirle conocimientos relacionados con la sexualidad según vaya creciendo. Aumentar las caricias, besos y los contactos físicos en general con su hija/o. Cuando crezca se le trate por un profesional el problema que pueda tener. Otro criterio:

3. Ha recibido Ud. información sobre el desarrollo de la sexualidad de su hija/o: $\mathrm{Si}$

4. Quién le ha brindado esa información:

Médica/o fisiatra que atiende a mi hija/o Neuróloga/o que atiende mi hija/o__ Técnica/o de fisioterapia___ Médica/o de la familia__

Psicóloga/o__ Otra madre o padre__ Un familiar__ Otra persona ¿cuál?

5. Considera Ud. suficiente la información recibida: Si__ No__ No sé__

6. El desarrollo de la sexualidad de su hija/o dependerá de su recuperación motora: Si__ No__ No sé__

7. El desarrollo de la sexualidad de su hija/o dependerá del aspecto físico que alcance: Si No No sé

8. El desarrollo de la sexualidad de su hija/o dependerá de su capacidad de tener erecciones, orgasmo u otra respuesta sexual: $\mathrm{Si} \_$No__ No sé_

9. La educación sexual para niñas y niños con parálisis cerebral: Siempre es necesaria__ No se debería hacer porque puede afectar a las/os niñas/os psicológicamente___ Solo es aconsejable para niñas/os con buen desarrollo psicomotor Puede exacerbar los instintos sexuales del niñas/os No sé que opinar

10. Considera Ud. necesario recibir información sobre la sexualidad de infantes con parálisis cerebral. Si___ No

Figura 1. Encuesta realizada a las madres de las niñas y los niños que se diagnosticaron con una parálisis cerebral.

Se desarrolla el presente estudio con las madres y los padres de las niñas y los niños diagnosticados con parálisis cerebral que asisten a la Clínica de Atención a Pacientes Cubanos del CIREN con el objetivo de conocer la visión que poseen sobre la sexualidad de sus hijas e hijos. Yo ---------------------- accedo a participar en la investigación después de haber sido informado que:

El ejercicio es de carácter estrictamente confidencial. La entrada al estudio es voluntaria y exenta de compromisos. El resultado del estudio no influirá en la rehabilitación de su hija/o. La no aceptación en la participación del estudio no influirá en los objetivos planteados para el ciclo rehabilitación que ha de recibir su hija/o.

Para que asi conste, firmo el presente documento a los días del mes de de

Firma de la madre o padre. Firma de encuestadora.

Figura 2. Acta de consentimiento Informado.

Del nivel empírico, también fue utilizada la técnica cualitativa de entrevista en profundidad, para indagar más a fondo la visión sobre temas de la sexualidad de madres y padres de infantes con parálisis cerebral (anexo 3). Esta modalidad de entrevista permite obtener una información más detallada sobre el tema investigado, ya que para conformar con claridad la idea objeto de análisis, se requiere volver una y otra vez sobre el mismo tema. La entrevista en profundidad permite acceder al conocimiento, las creencias, los rituales, la vida en sociedad o cultura, obteniendo datos en el propio lenguaje de los sujetos.
Tanto el cuestionario como la entrevista en profundidad fueron antecedidas por el consentimiento informado de cada persona, se realizó el estudio teniendo en cuenta las condiciones óptimas para su desarrollo, contando con el tiempo necesario para su ejecución y la privacidad requerida (anexo 4).

\section{Resultados}

Al inicio de la investigación se aplicó un cuestionario para abordar el tema desde una óptica exploratoria con las madres que conformaron la muestra, dirigido a indagar la visión que poseían sobre la sexualidad de sus hijas/os.

Las madres encuestadas refieren que sus niñas/os quizás pueden desarrollar una sexualidad gratificante una vez llegada la adultez. Expresan que no todos podrán desarrollar esta dimensión de la personalidad y fundamentan este argumento con la presencia de la enfermedad neurológica y la no recuperación física, lo cual podría interferir en el desarrollo de cualquier aspecto de la sexualidad.

Para lograr un desarrollo pleno de la sexualidad de sus hijas/os consideraron necesario realizarle el máximo de ejercicios para que mejoren sus condiciones físicas y también transmitirles conocimientos relacionados con este tema según vayan creciendo. Indistintamente, las madres encuestadas conceden importancia a los afectos, por lo tanto, aumentar las caricias, besos y contactos físicos en general con sus hijos/as puede ser considerado un aspecto importante para el desarrollo de la sexualidad. Todas las madres refieren que tratar con un profesional el problema que pueda presentarse cuando crezca puede ser una solución acertada.

Las madres informan que no cuentan con información suficiente y científicamente establecida sobre el desarrollo de la sexualidad infantil. Para conocer quién pudo facilitarla en algún momento, se exponen como opción personas que por su rol profesional deben tener mayor contacto con los infantes y con sus familiares. Entre ellos el médico fisiatra, neurólogo, fisioterapeuta, médico de la familia, psicólogo, algún familiar, otras madres o padres $u$ otras personas. Una de las madres expresa que la información la había recibido del psicólogo que atiende a la niña. Otras tres refieren que un familiar no vinculado directamente con los niños fue la fuente de información.

Las madres encuestadas no consideran suficiente la información recibida, informan que el desarrollo de la sexualidad de sus hijas/os dependerá de su recuperación motora, mientras que algunas de ellas no saben si su descendencia podría alcanzar logros con esta recuperación. Alegan que el desarrollo de la sexualidad dependerá del aspecto físico que alcancen. Todas las encuestadas informan que el desarrollo de la sexualidad de sus hijos/as dependerá de la capacidad de tener erección, orgasmo u otra respuesta sexual; este aspecto denota la falta de información que prevalece sobre los temas de sexualidad y discapacidad.

La generalidad de las madres expresan que la educación sexual de niñas/os con enfermedades neurológicas es necesaria y alegan que es aconsejable para niños/as con buen desarrollo psicomotor. Opinan que la educación de la sexualidad puede exacerbar los instintos sexuales del niño/a, por lo que es necesario recibir información sobre la sexualidad de infantes con enfermedades neurológicas. 
Una vez concluida la aplicación de la encuesta y el análisis de los resultados obtenidos en esta técnica, determinamos que resultaba necesario aplicar otra técnica que nos permitiera explorar más a fondo la visión sobre la sexualidad infantil que poseen las madres de niñas y niños diagnosticados con parálisis cerebral. Durante el desarrollo de esta fase del estudio se incorporaron los padres que acompañaban a sus hijos/as durante el proceso de restauración neurológica. Se aplicó la técnica de entrevista en profundidad a las madres y los padres de infantes con parálisis cerebral.

Según la cronología que se propuso en el guión de entrevista en profundidad se obtiene la siguiente información.

\section{Criterios de las madres y padres acerca de la sexualidad}

Las madres y padres entrevistados expresan que "los temas de la sexualidad no deben ser abordados con sus hijas/os porque se dificultaría hacerlo teniendo en cuenta la edad y el estado de salud de su descendencia. Expresan no tener toda la referencia para tratar los temas de la sexualidad infantil y no cuentan con la información de cuáles temas pudieran ser abordados con sus hijas/os. Reconocen durante la entrevista que hasta para ellos se agotan los recursos de información y no tienen las herramientas para abordar cualquier tema de la sexualidad con sus hijos/as".

Expresan madres y padres que "este no es el momento para abordar estos temas y sería mejor hacerlo cuando lleguen a la adolescencia o la juventud". "Estos temas no se tratan desde tan tempranas edades para no incentivar deseos y motivaciones que no puedan cumplirse". "La sexualidad es un tema para los adultos y no para las/os niñas/os". Expresan reiteradamente durante la entrevista que la sexualidad es un tema exclusivo para los adultos.

Madres y padres opinan que estos son temas que en su momento deben ser tratados por especialistas preparados en esta temática y también por las/os educadores en las escuelas y no por la familia que, la gran mayoría de las veces, evita abordar estos tópicos porque no le ven futuro a sus hijas/os socialmente, entonces mucho menos sexualmente.

Reiteradas veces durante la entrevista se escucharon argumentos como los siguientes: "sería imperdonable incentivar conductas desviadas en los niños", "me atrapa el miedo por tantas cosas, pero creo que moriría si mi hija se convierte en objeto de abuso sexual", "la sociedad suele ser muy exigente, las personas discapacitadas son rechazadas por los buenos y los malos se aprovechan", "preferiría no enseñarle cosas de su sexualidad, no permitiría que nadie se le acercara jamás", "no imaginaba que la autoestima y los autocuidados formaran parte de la sexualidad". Estos testimonios denotan la angustia e inseguridad que caracteriza la vivencia de estas familias en las que muchas veces la presencia de la discapacidad es sinónimo de profundo dolor. El desconocimiento de cualquier tema que no esté relacionado con la rehabilitación física y cognitiva es una constante de lo desconocido y el tecnicismo deja a un lado los afectos. En la mayoría de las entrevistas los temores también se vieron permeados por las posibles manifestaciones de la violencia que se puede presentar y, en el caso de la discapacidad, le conceden una predisposición casi inherente. Si bien la violencia trasciende fronteras y existen muchas formas de manifestarla constituyendo un problema social y de salud, pueden ser evitados disímiles acontecimientos de este tipo tan solo con una correcta orientación del tema favorecido con una adecuada educación de la sexualidad.

Existe preocupación sobre qué deben saber concretamente para preparar a sus hijas/os para el futuro. Esta misma preocupación la expresan para el momento del enamoramiento y la selección de la pareja, temiendo que utilicen a sus hijas/os como objeto sexual donde no medien sentimientos y valores. Toda esta vivencia la expresan permeada de mitos, prejuicios y tabúes, estigmatizando no solo la conducta de las niñas y los niños, sino también la de todos aquellos que los rodean.

Ambiente escolar y sociocultural como facilitador de la
educación sexual

Durante la aplicación de la entrevista pudimos conocer a madres y padres que expresan no conocer las alternativas presentes en la escuela para abordar el tema de la sexualidad, lo cual no significa, aclarado en todos los casos, que no existan estas alternativas. Algunas de las niñas de estas parejas asisten a las vías no formales y se preparan para transitar a la enseñanza primaria; las madres de este grupo informan que en determinados encuentros en los que han participado se ha abordado el tema de la sexualidad, referido fundamentalmente al género, haciendo énfasis en la propuesta de actuaciones para las niñas y niños en la proyección de los diferentes roles. La mayoría de los infantes asisten a las instituciones de salud pública y allí reciben atención ambulatoria desde el punto de vista educativo y se tratan por parte de las maestras ambulatorias temas escolares vinculados con la estimulación temprana. Solo dos menores no asisten a alguna alternativa para la educación por no tener la edad requerida. En todos los casos informan que no conocen la existencia de programas en las diferentes instituciones a las que asisten para la educación de la sexualidad de sus hijos/as.

Madres y padres expresan que sus niñas/os logran integrarse en los distintos contextos que la comunidad facilita para la interacción social. La mayoría asiste de manera irregular a parques interactuando con coetáneos. De la misma forma, asisten a casas de cultura, centros recreativos, instituciones de salud e iglesias, y realizan visitas a familiares que no conviven con los menores.

Los temas relacionados con la sexualidad no constituyen interés principal de la familia para contribuir al desarrollo personal de sus hijas/os. No es un tema priorizado como lo es la rehabilitación física. Refieren que todavía son pequeños para exponerse a estos contextos y alegan que "nuestra sociedad no está preparada para convivir con la diferencia".

\section{Composición y relaciones intrafamiliares}

En todos los núcleos familiares estudiados se constata la presencia de una niña o niño con diagnóstico de parálisis cerebral. La mayoría de las/os infantes conviven con sus madres y padres, además de otros miembros de la familia, como hermanos, que no presentan ninguna discapacidad, abuelos maternos y/o paternos, siendo más numerosa la representación de abuelos maternos.

La mayoría de las madres y padres informan que las relaciones intrafamiliares y la comunicación fluyen adecua- 
damente, ya que son sentidos los afectos y prevalece un ambiente de respeto y atención con la posibilidad para que cada miembro de la familia pueda expresarse, también recibir criterios de los otros de manera asertiva, sin olvidar que existen costumbres y tradiciones familiares que van pasando de generación en generación, como paradigmas que se detienen en el tiempo y se vuelven inviolables.

Esta referencia no es válida en todos los casos, ya que, aunque pocos, existe un grupo de madres y padres que durante la entrevista expresaron que las relaciones entre los miembros de su familia no eran las mejores, por lo que no cuentan con satisfactorias redes de apoyo. Muchas veces se muestran desentendidos con cualquier aspecto relacionado con la cooperación necesaria para el cuidado y atención de niñas y niños. En este grupo se dificulta la dinámica para las buenas relaciones entre sus miembros.

En este aspecto, se indaga sobre cómo es o puede ser tratado el tema de la sexualidad a partir del buen o deficiente establecimiento de las relaciones intrafamiliares y la comunicación. En todos los casos refieren que la principal preocupación de las familias no es precisamente el desarrollo psicosexual de sus hijos/as, sino, sin duda, la recuperación de las capacidades físicas y psíquicas. Sin embargo, en la medida en que se desarrollaba la entrevista se podía observar como aumentaba la motivación por el tema y surgieron preguntas que denotaban preocupación por lo relacionado con los temas de la sexualidad.

Términos que utilizan madres y padres para abordar el tema de la sexualidad

Madres y padres expresan que conocen algunos de estos términos relacionados con la sexualidad humana y los fundamentan a partir de las experiencias vividas. Asocian la sexualidad con algunas de las vivencias personales, vinculadas fundamentalmente con la respuesta sexual biológica y la capacidad de formar pareja erótica. Se constató que existen vacíos en el conocimiento sobre los órganos sexuales femeninos y masculinos.

Otro de los elementos mencionados son los referentes al esquema corporal, en el cual también pudimos corroborar que son omitidos determinados segmentos corporales, fundamentalmente los que se relacionan con los miembros superiores e inferiores.

Madres y padres hacen referencia a la reproducción y el placer erótico con mucha preocupación. Expresan que el desarrollo de la sexualidad de sus hijos/as debe subordinarse únicamente a la capacidad de tener erección, orgasmo u otra respuesta sexual. Asumen que, ante la discapacidad, las emociones y los sentimientos no son visibles para el mundo que les rodea.

Se dificulta encontrar otros referentes de esta temática durante la entrevista, quedando excluido uno de los aspectos distintivos de las funciones vitales de la sexualidad. Si bien la función reproductiva y el placer erótico resultan términos muy conocidos, por ser intermediarios muchas veces de las diversas vivencias afectivas que expresan los seres humanos, no podría obviarse un tercer elemento que conforma las funciones de la sexualidad, como lo es la comunicación. Se manifiesta en todos los momentos existenciales en los que se presentan las relaciones con otras personas, en el contexto de la familia y la sociedad, por lo tanto, también en los espacios de la pareja y de cada sujeto.

Preocupaciones fundamentales de madres y padres relacionadas con la sexualidad de sus hijos/as

Durante el desarrollo de la entrevista resultó visible la ansiedad con que fueron abordados los tópicos del cuestionario. Las preocupaciones fundamentales relacionadas con la sexualidad de sus hijas/os fueron:

- Abordaje del tema de la sexualidad a partir de la discapacidad.

- Falta de información para afrontar los temas de la sexualidad infantil.

- Proyección del futuro de sus hijas/os a partir de la discapacidad y el conocimiento de la sexualidad.

\section{Valoración de situaciones existenciales}

Para madres y padres de niñas y niños diagnosticados con parálisis cerebral es una preocupación latente la recuperación física-motora y cognitiva. Consideran que el desarrollo de la sexualidad es una posibilidad exclusiva para aquellos que establezcan la diferencia con las características propias del diagnóstico que presentan sus hijas/os. Subordinan el enriquecimiento de esta importante dimensión de la personalidad a la recuperación física que, a su vez, se sostiene a través del proceso de rehabilitación que se establece en tiempo ilimitado, con pronósticos muchas veces reservado.

Se considera la discapacidad como limitación para expresar la sexualidad, también como una de las situaciones existenciales más contradictorias, porque se proyecta como un equivalente al placer erótico asociado a las limitaciones para sostener la satisfacción del ser hombre o mujer con una discapacidad. No perciben la sexualidad como una parte indisoluble de la personalidad que se complementa en todas las dimensiones con la presencia de derechos y deberes, decisiones, necesidades y proyecciones para obtener una vida plena.

Se empleó el método de la observación para establecer el primer punto de vista que permitió conformar el presente estudio a partir del reconocimiento del vínculo que, en el contexto de la rehabilitación, establecen niños y niñas con madres, padres y terapeutas.

De esta manera, se pudo conocer que la interacción en el contexto terapéutico entre las madres y padres con sus hijas/os se vuelve más técnico que afectivo, ya que constantemente insisten en la correcta postura de los miembros superiores e inferiores, corrigen la marcha y el lenguaje. Desde las primeras interacciones de los terapeutas con madres $y$ padres se pudo constatar que no se favorece la independencia con sus hijos/as. Facilitan la ayuda asistida para que realicen cualquier actividad de la vida diaria, aunque las niñas y niños puedan desarrollarse independientemente en algunas de estas actividades.

Los familiares expresan desconfianza y muchos miedos porque sus niñas/os son muy pequeños/as. Temen que sufran alguna caída por no encontrarse protegidos por ellos y refieren la necesidad de la atención directa de otras personas para que puedan cumplir con algunas de las actividades programadas.

Los afectos físicos y verbales no son totalmente visibles. No fueron frecuentes las palabras 0 frases entre 
madre/padre - hijo/a; muchas veces la voz está ausente y realizan el acompañamiento con una expresión del rostro que refleja angustia y desconfianza. Como expresión de caricia, pasan la mano por la cabeza, el rostro y las extremidades. Frente a la discapacidad las madres y los padres reflejan falta de recursos y de estabilidad emocional, mostrando una ilusión poco objetiva por una pronta recuperación.

Las niñas y los niños diagnosticados con parálisis cerebral aceptaron a las terapeutas con la finalidad de recibir la rehabilitación por la cual se encontraban en el CIREN. Algunas de las niñas y los niños necesitaron de algún tiempo durante la primera semana para lograr la separación de madres y/o padres que les acompañaban, con el fin de trabajar en los locales establecidos, coincidiendo con aquellas/os infantes que nunca habían recibido rehabilitación en nuestra institución; también coincide con las edades más pequeñas de este grupo de infantes.

Durante la aplicación del cuestionario, se observó que las madres necesitaron algún tiempo para dar respuesta a las preguntas y realizaban comentarios con la encuestadora alegando sentirse extrañadas por abordar el tema de la sexualidad de sus hijas/os. Hubo madres que durante y después de la aplicación del cuestionario no pronunciaron ni una sola palabra y miraban a la encuestadora con desconfianza.

En el desarrollo de la entrevista en profundidad se pudo observar mayor interés por el tema tratado. En este momento del estudio se habían incorporado los padres y de la dinámica de la entrevista fluía polémica, pero con la presencia del dialogo. Se pudo constatar la presencia de pausas a la hora de que madres y padres expresaban sus criterios sobre la sexualidad. Brazos cruzados durante el intercambio, rubor del rostro de las madres y los padres durante la exposición de sus argumentos, acompañado por silencios prolongados.

Expresan las pautas de la conducta que han adoptado para con sus hijos/as, regidas por prejuicios que llegan a sus saberes por las tradiciones familiares y las conductas que la sociedad norma de manera desacertada.

Como un elemento interesante se destaca que, después de concluidas las sesiones de entrevistas con las madres y los padres, se acercaron nuevamente a la entrevistadora con nuevas interrogantes relacionadas con la sexualidad, alegando que "quizás podría ser tarde para enterarse que también desconocían muchas cosas de su sexualidad como hombres y mujeres, después de haber vivido una parte importante de sus vidas". Otra de las expresiones repetidas fue "nos gustaría intentar enmendar los desconocimientos sobre la sexualidad que expresamos y de esta manera podremos ayudar mejor a nuestros hijos/as".

Necesidades básicas de aprendizaje identificadas en las madres y los padres sobre temas de la sexualidad infantil

Durante el estudio se aplicaron diferentes técnicas a madres y padres, las que permitieron determinar el estado actual de las necesidades básicas de aprendizajes sobre temas de la sexualidad infantil.

Se identificó que:

Existe desconocimiento sobre los temas de la sexualidad infantil por parte de las madres y los padres de las niñas y niños diagnosticados con parálisis cerebral. No le conceden la importancia necesaria al desarrollo de la sexualidad de sus hijas e hijos y relacionan la sexualidad de las personas que presentan alguna discapacidad con la respuesta sexual biológica, la capacidad de formar pareja erótica y con algunas manifestaciones de violencia. Excluyen otros aspectos como los afectos que se entregan y reciben desde el mismo nacimiento y el cuidado de una autoestima positiva por no considerarlos parte de la sexualidad.

Los conocimientos que poseen sobre la sexualidad y la discapacidad están permeados de mitos, tabúes y prejuicios que se incorporan a través de las tradiciones familiares, las cuales favorecen conductas de sobreprotección. Por tanto, la ausencia de información sobre esta temática en los adultos constituye vacíos que ocupan el saber de la infancia. Existe poca preparación en la esfera de la sexualidad infantil desde el rol de madres y padres. Durante el desarrollo del estudio, se pudo constatar que no poseen los conocimientos necesarios para acompañar a su descendencia en el proceso desarrollador de la sexualidad desde la niñez.

El estado deseado permite enfocar los objetivos que a mediano y largo plazo se desea obtener proyectándose de la siguiente manera:

- Ofrecer conocimientos sobre los temas de la sexualidad infantil a madres y padres de niñas y niños diagnosticados con parálisis cerebral.

- Reflexionar sobre los mitos, tabúes y prejuicios relacionados con la discapacidad y la sexualidad infantil.

- Ofrecer durante un proceso desarrollador conocimientos sobre las características psicosexuales de la infancia en sus diferentes etapas.

- Ofrecer herramientas que favorezcan la adquisición de conocimientos, destrezas y habilidades que permitan la formación de una sexualidad sana, desprejuiciada, mediante un proceso de educación y comunicación desarrollador.

Propuesta de acciones socioeducativas que contribuyan a la educación sobre temas de la sexualidad infantil

Las madres y los padres constituyen ejemplo y conocimiento para las nuevas generaciones. Incorporar los temas de la sexualidad para las personas que presentan alguna discapacidad en su proceso de neurorestauración podría significar un salto verdadero ante las falsas creencias que estigmatizan las vivencias de estas personas.

Las acciones socioeducativas, como proceso organizado, formalizado y orientado a la obtención de una meta claramente establecida, tienen enfoques metodológicos para su intervención.

Se establece como proceso formativo, ya que favorece el acceso al conocimiento y el desarrollo de competencias y habilidades. Facilita la transformación de actitudes y comportamientos, el aprendizaje activo, la construcción del saber y posibilita el desarrollo. Desde lo cognitivo, proporciona adquisición de conocimientos, destrezas intelectuales y desarrollo de habilidades (Gener et al., 1998).

Otro de los enfoques metodológicos para la intervención socioeducativa es el cultural y recreativo, el cual favorece la creatividad, el enriquecimiento personal a través del arte y la creación cultural. Es de acceso a bienes comunitarios, porque beneficia la participación, la cooperación y la solución conjunta de problemas colectivos. Se expresa además como lúdico por su utilidad en el desarrollo físico, mental y personal mediante el 
juego, y es participativo por su acción en la cooperación y solución conjunta de problemas colectivos (Gener et al., 1998).

Los fines deseables de las técnicas de intervención socioeducativas van encaminados a desarrollar una cultura de trabajo cooperativo y participante. Permite que las personas implicadas pasen por un proceso de empoderamiento y aprendizaje social. Promueve el desarrollo de habilidades de interacción social al propiciar la participación, desempeñando diferentes roles durante las labores propias de cada actividad. La intervención socioeducativa motiva a los participantes a desarrollar una identificación positiva con los fines de la interacción, haciendo la forma de trabajo más congruente con la realidad social. Estimula el espíritu de equipo y el sentimiento de pertenencia en una comunidad (Gener et al., 1998).

La sexualidad infantil de niñas y niños que presentan alguna discapacidad implica educación acertada, ya que las nuevas generaciones necesitan este conocimiento desde edades tempranas para evitar ignorancias que empobrecen el futuro. Enriquecer el conocimiento de madres y padres sobre temas de la sexualidad infantil permite favorecer el disfrute de una sexualidad sana y desprejuiciada para sus hijas/os.

\section{Acciones socioeducativas}

- Seleccionar el personal adecuado para desarrollar dinámicas grupales con fines educativos sobre temas de la sexualidad infantil.

- Capacitar el personal que desarrollará las dinámicas grupales con madres y padres sobre temas de la sexualidad infantil, para lo cual se tendrá en cuenta:

- Encauzar los objetivos de la educación sexual en las distintas etapas del desarrollo de la personalidad.

- $\quad$ Favorecer la reflexión sobre mitos, prejuicios y tabúes relacionados con la sexualidad y la discapacidad.

- Trabajar con la familia como eje fundamental en el desarrollo de la sexualidad infantil.

- Desarrollar habilidades sobre endosexualidad con la familia de niñas y niños con parálisis cerebral.

- Orientar a las madres y padres sobre los temas del abuso sexual como manifestación de la violencia, como problema social y de salud.

- Desarrollar habilidades sociales en los sujetos objeto de estudio, haciendo énfasis en autoestima, autocuidado, asertividad y modos de afrontamiento como referentes necesarios para el tratamiento de los temas de la sexualidad.

- Coordinar con la dirección de la clínica el horario y el espacio físico para desarrollar las dinámicas grupales con las madres y los padres.

- Planificar la distribución de los temas a tratar durante el ciclo terapéutico para garantizar la asistencia de las madres y los padres a cada encuentro.

- Evaluar de manera sistemática todo el proceso de aplicación de las acciones propuestas para las madres y padres de infantes con parálisis cerebral.

Se proponen los siguientes indicadores para evaluar de forma sistemática la efectividad de dichas acciones.
Indicadores para la evaluación posterior de la efectividad de las acciones

- Conocimientos sobre la sexualidad infantil.

Se facilitará la comprobación de los conocimientos sobre sexualidad infantil y discapacidad a través de las dinámicas grupales una vez concluido el ciclo de trabajo con todas las madres y los padres.

- Temas asociados a la sexualidad infantil y la discapacidad. Se aplicará un cuestionario anónimo a todas las madres y padres para evaluar el aprendizaje sobre los temas tratados durante el ciclo de trabajo.

- Consejería y orientación socioeducativa.

Se verificará la creación de este espacio para que madres y padres tengan la opción de abordar siempre que lo deseen los temas tratados durante el ciclo de trabajo.

\section{Discusión}

Los infantes que presentan alguna discapacidad deben convivir en un ambiente donde prevalezcan los afectos, la comunicación y el respeto. En este sentido, la familia juega un papel determinante (López Sánchez, 2005). La forma en que se comienza a expresar la sexualidad en la infancia condicionará como el niño y la niña pueden vivir su sexualidad en otras edades.

Expresa Félix López que los resultados de las escasas investigaciones realizadas en este campo siempre han sido limitados. Por satisfacer su curiosidad, por conocer las diferencias con el otro sexo, por imitar a los adultos, por diversión o por descubrir su propio placer, los niños y niñas cuentan con una vida sexual más variada de lo que podría pensarse (Fuertes y López Sánchez, 1997).

En todas las edades existen intereses $y$ comportamientos sexuales. Cada edad es diferente y se vive de forma distinta. "Las niñas y los niños pequeños no solo tienen necesidades biológicas: comer, estar limpios, sino también sociales, afectivas y sexuales". "La sexualidad no está muy diferenciada de otros sentimientos de placer, bienestar y seguridad en las primeras edades" (López Sánchez, 2002).

Los prejuicios y tabúes que han acompañado a la sexualidad a lo largo del tiempo y en diferentes culturas, así como su carácter íntimo y privado hacen que muchos la perciban como algo que no debe ser indagado y que se debe dejar al libre albedrío. Este criterio toma más fuerza con la infancia, ya que incluso hay quienes niegan su existencia en tal etapa, consideran como perversiones sus manifestaciones y neutralizan los intereses infantiles en ese sentido (López Sánchez, 2002).

Aunque algunos aspectos de la sexualidad infantil, como la adquisición de la identidad sexual y el rol de género, han sido muy estudiados, otros como las conductas sexuales infantiles han sido y siguen siendo muy poco investigados. Las razones de esta ausencia son numerosas, se destacan dos de ellas: encontrarnos en una cultura que niega la existencia de la sexualidad infantil por considerar peligrosas sus manifestaciones y las dificultades éticas para el estudio de manera experimental, a través de observaciones o de preguntas directas a los menores (Fernández, 2000).

Son numerosos los prejuicios, mitos y tabúes que estigmatizan las conductas hacia estas personas, actitud que afectará significativamente su sexualidad. En el plano personal 
se pueden presentar alteraciones, muchas veces consecuencia de estos mitos y prejuicios, que también menoscaban la sexualidad de los discapacitados y que pueden ser el origen de alguna disfunción sexual $u$ otras insatisfacciones recurrentes (Torres Marrero, 2008).

Frecuentemente, la sexualidad de las personas con discapacidad se considera como limitada. Se pueden presentar trastornos más o menos severos de la respuesta sexual o problemas físicos, sensoriales o mentales; sin embargo, sus derechos, responsabilidades y expresiones sexuales son iguales que las del resto de la población. La solución no es buscar las diferencias, sino comprenderlas y obtener el máximo disfrute y plenitud personal y social con las capacidades residuales que se tengan.

A través del lenguaje, el adulto otorga un significado al cuerpo y sus funciones, expresando aceptación o rechazo. La sexualidad comprende todos los factores: lo psicológico, lo biológico y lo sociocultural, por lo que, en la medida en que el hombre aprende y se desarrolla como ser sexuado, establece también sus vínculos y afrontamientos con sus entornos, en los cuales puede desarrollar concepciones, estilos y modos de vida - adecuados o no- que influirán en sus afrontamientos y salud (Ortega Bravo, 2009).

Cuando se hace presente la discapacidad en un hijo, los padres asumen diferentes posiciones, en lo que incidirán factores situacionales y contextuales que se visualizan en cada familia. Las reacciones están estrechamente vinculadas con la dinámica del hogar, aun antes de que surja la discapacidad del hijo (Castro, 1995).

La aparición de la discapacidad condiciona ajustes en la estrategia de vida de cada familia. Estudiosos del tema plantean que se manifiestan conflictos tendentes a culparse por lo que ocurre, muchas veces recae en la madre como respuesta a los estereotipos existentes en cualquier sociedad, lo cual favorece la creación de un sentido distorsionado de las responsabilidades de cada miembro de la familia. (Castro, 1995)

Suelen evitar los ensayos para que sus hijos no cometan errores y desconocen muchas veces la etapa de desarrollo en la que se encuentran, creando a su vez otros problemas asociados con la discapacidad, denotando falta de recursos y estabilidad emocional para enfrentar la situación (Castro, 1995).

"La educación a la familia en torno a la sexualidad contribuye a la adquisición de conocimientos, desarrolla actitudes y convicciones, estimula intereses y consolida motivaciones. Facilita que los padres tengan una concepción humanista y científica de la sexualidad y su educación" (Ares Muzio, 2008).

Las madres y padres son paradigmas incalculables para sus hijas e hijos. El estrés de la vida cotidiana es utilizado muchas veces por los adultos para justificar las malas actuaciones en la educación de niñas y niños, convirtiéndose casi siempre en protagonistas o espectadores de ejemplos desafortunados, que llegan a ser visualizados por los más pequeños $y$, por tanto, los asumen como modelos a seguir de forma confiable.

Las personas con discapacidad no deben discriminarse por ser diferentes. Las niñas y los niños pueden expresar su sexualidad de forma diversa y también personalizada. En esta etapa de la vida, la infancia necesita aceptación y acompañamiento para el éxito en su crecimiento y formación como seres humanos de nuestro tiempo.

Las definiciones conceptuales que sostienen el presente estudio permitieron embridar el contenido de la información que facilitaron las madres y los padres objeto de estudio. Dimensionar el análisis de la información facilitó la proyección de la propuesta de las acciones socioeducativas.

Percepción social: Se analizará a través de la exploración del conocimiento sobre temas de la sexualidad infantil de las madres y los padres implicados en el estudio, apoyados en la siguiente postura epistemológica sobre conocimiento.

Conocimiento: Proceso mediante el cual los seres humanos incorporan ideas, adquieren saberes específicos, asimilan conceptos acerca de los fenómenos reales y van comprendiendo el mundo que los rodea

El análisis se realizará a través de:

- Criterios de madres y padres sobre el tema, con los cuales son expresados sus saberes acerca de la sexualidad, con la presencia de mitos, prejuicios y tabúes que perviven en las personas entrevistadas.

- Los silencios sobre el tema durante el desarrollo de la investigación.

- Expresión de los conocimientos de madres y padres sobre la educación de la sexualidad de sus hijas e hijos, a partir del acceso a las alternativas para la educación de la sexualidad, no solo en los espacios escolares sino también en la comunidad.

- Forma en que las madres y los padres nominalizan los términos sobre sexualidad en la comunicación con sus hijas/os, que puede coincidir o no con lo establecido por la literatura científica.

- La referencia que tienen madres y padres sobre las funciones vitales de la sexualidad.

- Aprendizaje sociocultural de madres y padres y su proyección en la formación de valores que favorezcan el desarrollo de una sexualidad sana y feliz para sus hijas/os.

Socialización: Se explorará a partir de los principales espacios en los que se insertan las madres y los padres que forman parte de la investigación.

El análisis se realizará a través de:

- La proyección del proceso de comunicación como una condición necesaria para la existencia de las interrelaciones que establecen las madres y padres en el contexto de las dimensiones existenciales.

- La socialización que establecen madres y padres sobre conflictos familiares asociados a la discapacidad de sus hijas/os.

- Expresión de los afrontamientos de madres y padres ante las preocupaciones existentes asociadas a la sexualidad y la discapacidad.

\section{Conclusiones}

Se diseñó un plan de acciones socioeducativas para la educación sobre temas de la sexualidad a las madres y los padres de infantes con parálisis cerebral que asisten al CIREN. Las acciones socioeducativas permitirán la incorporación de herramientas para la sistematización de conocimientos específicos sobre la sexualidad infantil.

El diagnóstico mostró que las madres y los padres estudiados poseen conocimientos insuficientes sobre los temas de la sexualidad infantil, los saberes están permeados de mitos y prejuicios relacionados con la discapacidad y la sexualidad. No 
conciben la educación de la sexualidad como un proceso necesario para el desarrollo personal de las niñas y los niños diagnosticados con parálisis cerebral.

\section{Recomendaciones}

- Aplicar las acciones socioeducativas y validarlas en un periodo de tiempo de 6 meses a un año.

- Incluir en el estudio otros diagnósticos de niñas y niños que asisten al CIREN para recibir el Programa de Neurorehabilitación Multifactorial Intensivo.

- Emplear los resultados en la elaboración de un programa para madres y padres sobre los temas de la sexualidad infantil en el CIREN.

\section{Referencias}

Ares Muzio P. (2008). Conferencia "La intervención familiar en las actuales realidades sociales". III Congreso Panamericano de Salud Mental Infanto Juvenil. Reunión de la Sección de Clasificación de la Asociación Mundial de Psiquiatría, La Habana.

Castro P. L. (1995). "Familia, sexualidad y discapacidad", en Selección de materiales del VII Congreso Latinoamericano de Sexología y Educación Sexual, Ed. Ciencia y Técnica, La Habana.

Fernández, J. (Ed.) (2000). Intervención en los ámbitos de la sexología y la generología. Madrid: Pirámide.

Fuertes, A. \& Sánchez, L. (1997): Aproximaciones al estudio de la sexualidad. Salamanca: Amarú.

Giner, S., Lamo de Espinosa, E. \& Torres C. (1998). Diccionario de Sociología. Ciencias Sociales. Alcanza Editorial.

Gil-Almira, A., Cardero-Pacheco, S. (2003). Consideraciones sobre la sexualidad en personas con discapacidad físico motora. MEDISAN, 7(2):49-57.

González, A., Castellanos, B. (2003). Sexualidad y Géneros. Alternativas para su educación ante los retos del siglo XXI. . La Habana: Editorial científico técnica.

López- Sánchez, F. (2005). "La educación sexual de los hijos". España: Pirámide,

López Sánchez, F. (2002). Sexo y afecto en personas con discapacidad. Ed. Biblioteca Nueva.

Martínez, M. (2006). La investigación cualitativa (síntesis conceptual). Revista IIPSI 9 (1), 123-146.

Ortega-Bravo, A. J. (2009). "Estrés, Salud y Sexualidad". La Habana: Capitán San Luís.

Rodríguez-Gómez, G., Gil- Flores, J., García-Flores, E. (2002). Metodología de la investigación cualitativa. Ed. PROGRAF.

Torres- Marrero, L. (2008). Visión materna de la sexualidad de niños con Parálisis Cerebral (Tesis presentada en opción del título de especialista de 1er grado en medicina física y rehabilitación). La Habana. 